\title{
A Brief Discussion of the Liability Attribution Basis of Hyperlink Infringement
}

\author{
Zhe Wang \\ Fuzhou University of International Studies and Trade, Fuzhou, China
}

Keywords: hyperlink, infringement, liability attribution principle

\begin{abstract}
Hyperlink technology is an important information communication technology in the Internet era. With the rapid development of the network economy, the application of the hyperlink technology in various types is becoming increasingly frequent, and website operators are paying more attention to the ownership attribution of their network information resources and the protection of commercial interests as well, thus leading to a large number of disputes on intellectual properties. This paper intends to set about different types of hyperlink technologies, combined with the existing agreed research achievements of the scholars, so as to restate the liability attribution principle and identification standard of the hyperlink infringement behaviors starting from typical cases, with an expectation to provide beneficial countermeasures for solving related infringement disputes for website operators and promote the network hyperlink technology to have a better communication and development in the public domain.
\end{abstract}

\section{Introduction}

According to the paraphrasing of Black's Law Dictionary, hyperlink technology refers to the information acquisition technology which can establish links between different parts of the same page or different pages through text, graph, label, command and other mediums, so that users can achieve free access between different pages and columns. It has been regarded as an "intellectual property" in Europe and the America.

\subsection{Categories of hyperlink}

According to different hyperlink technologies, hyperlink is mainly divided into three categories:

HREF link. Generally speaking, all hyperlinks belong to HREF link. However, according to the different subjective intents and link modes of the link setter(hereafter named as setter for short), HREF link can be divided into homepage link and page link(or named as “deep link"). Just as its name implies, as to homepage link, setter guides the website visitors(hereafter named as visitors) to the homepage of another website, and its address field still reserves the website of the linked website. Its legal risk is relatively low. However, the deep link we discussed in the intellectual property field means that the setter reaches the web interface it wants to use directly bypassing the homepage of the linked website, and the address bar still shows the address of the setter. Undoubtedly, visitors will be unable to distinguish the real originator of the website content and get confused, thus affecting the benefits of the original website operator.

Embedding link. In embedding link, the setter displays the content of the linked website in its own web page through kink, with the address bar displaying the website of the setter as well, so that visitors “can't perceive” that the content of the web page is originated from other web pages.

Frame link. Both embedding link and frame kink belong to its branch. At its most basic level, it is also one of the embedding links. Its technology characteristic lies in dividing the window of the linked website into several small windows, so that visitors are also unable to find out the real address of their browsing web page.

All the above three hyperlink categories will probably give rise to legal disputes. Especially the deep link, the behavior of neglecting the permission of the linked website is nothing else but setting up a toll station in front of others' private gardens by force to get profits without getting the permission in the real world. However, many of the commercial interests in the virtual world are often not directly reflected by the amount of click rates. 


\subsection{Reasons for hyperlink generating disputes}

Generally speaking, the linked website appeals the arbitrary setter to court mainly based on the following three reasons:

The main source of revenue for a website is advertising revenue. In the network world, advertising revenue has direct relationship to the page view and click rate of the visitors. If the setter adopts the hyperlink technology deliberately to skip the true website of the linked page, it will result in a direct decrease in the number of page view and click rate of the linked website, which will directly affect the revenue.

When some websites set links, they usually use "reverse passing-off", which means to reserve their own domain names on the linked website, so as to mislead visitors to have an illusion that the web pages of the setters have a certain association with the linked website. As a result, once the website of the setter has any bad evaluation, these negative contents will probably be exerted on the linked web page, thus damaging the reputation of the linked website. For example, in order to publicize and promote, one civil IELTS training organization links many parts of its own website to the official page of IELTS China arbitrarily, with an expectation to show its authority. However, if this organization suffers from credit damage because of bad management in the future, the reputation of British IELTS Training Center will certainly be affected as well.

The behavior of setting hyperlink without authority by the setter is obviously full of commercial hostility and has the suspicion of "thumbing a lift" for unfair competition. Meanwhile, it also infringes the information network communication right of the original ownership of the website.

\section{Empirical Analysis: Liability Attribution Principle of the Link Infringement}

As to the liability attribution principle of the hyperlink infringement, the domestic academic world and practice field have basically formed the consensus on the principle of liability for fault, which is reflected in the following two typical cases.

\subsection{Beijing Seven Color Rainbow Advertising Co., Ltd. vs. Zhejiang Taobao Network Co., Ltd. copyright dispute case}

In 2011, in Beijing Seven Color Rainbow Advertising Co., Ltd.(hereafter named as Seven Color Rainbow Company) vs. Zhejiang Taobao Network Co., Ltd. (hereafter named as Taobao Company) copyright dispute case, the defendant Taobao Company has loaded 18 pictures of the rights of Seven Color Rainbow Company in its sub-pages including “Taobao Portal-Women·Marriage” . Meanwhile, if the Taobao Portal-Women·Marriage column of the defendant is clicked, the website IP address column displays as “http: //taobao.onlylady.com”. The accuser-Seven Color Rainbow Company considers that Baobao Company constitutes an infringement for spreading its production without permission, thus suing it to court.

The pleading excuse of the defendant Taobao Company is: it only links the page content of the third-party website through iframe, namely, its website content is provided by the third-party website. In addition, after being sued, it has already disconnected the corresponding link, so Taobao Company should be affirmed to constitute no infringement according to the "safe harbor rules".

The court held that the decision-making power on setting the content, column and section of the involved web page was possessed by the defendant Taobao Company. Therefore, Taobao Company shall have the examination duty to the legality of the web page content in its channel. Because of its negligence in fulfilling this obligation, it shall bear corresponding responsibility for the infringement. Therefore, Taobao Company was ordered to suspend infringement and compensate the loss.

In the case, the court made a detailed interpretation to the legal attribute of iframe link, further affirmed the determination basis of "user standard theory", stated the exceptions of "safe harbor rules" and subjective fault behaviors of the defendant. Retrospecting the judgment of the case, despite the court eliminated the application of "safe harbor rules" with "user standard theory", the basis of the judgment that the defendant assuming the legal responsibility still couldn't do without 
the affirmation of the subjective viciousness of the defendant. In other words, the defendant possessed complete decision-making power to the settings of the content, column and section of the involved web page, but its behavior of setting the website of the accuser as its own has not met the requirements of subjectively unknown or knowable, thus its subjective viciousness can be presumed. Therefore, the defendant indeed adopted iframe link to provide content with the name of link. Its pleading excuse is naturally untenable.

\subsection{Infringement dispute case of QvodPlayer information network communication right}

There was another typical case in 2013: China Film Co., Ltd., Film Marketing and Scheming Branch(hereafter named as China Film Marketing Branch) vs QvodPlayer Information Network(hereafter named as QvodPlayer), which has been elected as one of the top ten typical intellectual property cases in Guangdong. The accuser China Film Marketing Branch sued to court and stated that the software subordinated to QvodPlayer has infringed the information network communication right of its productions. The accuser stated that through putting in key words in the software search box of the QvodPlayer player subordinated to the defendant, it would guide to the third-party search website, which would display the search result of all resources. These resources are all complete videos of involved films. Meanwhile, the video playing website would be linked as well according to the above search result and the involved films can be clicked to play.

The court held that: “although the search engine integration proposed by the defendant doesn't search or link the productions involved in the infringement directly, it guides the content required by the user to the third-party search website, which not only limits the search object to the document of a certain category, but also restricts the search scope into a specific website. Therefore, it can be affirmed that the defendant and the search website jointly perform the behavior of searching and linking the productions involved in the infringement. The defendant is subjectively at fault. Therefore, the court ordered the defendant to stop the infringement and compensate the loss to the accuser."

Different from the prior case, although QvodPlayer company doesn't adopt iframe link technology to "occupy" the content of the linked website, the search link service provided by QvodPlayer software also has the condition of subjectively unknown or knowable without the consent of the linked website-despite QvodPlayer software doesn't conceal the domain name of China Film Company after providing the link search for free, subjectively, its behavior of skipping the home page of China Film Company to the play site of the film directly, combined with the film watching habits of ordinary film watchers, could not eliminate all its deliberations of excepting the film watchers to have the misunderstanding that the film belongs to QvodPlayer. Therefore, QvodPlayer still has to assume the legal responsibility for its own fault.

\section{Conclusion}

In practice, many websites will "exchange" friendly links with a great amount of websites in order to "huddle together for warmth", "join hands in strength" and increase their click rates. For example, an intellectual property company in Guangzhou has over 20 friendly links in the lower part of its home page. Why does such a condition have no legal dispute? The reason is self-evident-the visitors can clearly see the domain name and website transformation in clicking the friendly link. Apparently, they will not mix the website content and material ownership. On the other hand, such a behavior of "exchanging" friendly link has been agreed by the two parties in advance, thus being different from the arbitrary using, which might cause complicated legal consequences.

However, the intellectual property dispute triggered by hyperlink in this paper concentrates mainly on the discussion on the deep links, such as: bypassing the home page of the linked website and reserving its own domain name without the content of the linked website, or the deliberate "thumb a lift" behavior. 


\section{References}

[1] Li Zhuo. Analysis of Intellectual Property Infringement of Network Hyperlink [J], Journal of Intelligence. 2004(5).

[2] Jiao Wenming. Legal Regulations of Hyperlink Infringement [J]. Journal of Nanjing University. 2001(5).

[3] Hei Xiaobing. Study on Network Link Infringement Legal Problems [J]. Science-Technology and Law. 2011(4).

[4] Yang Dong. Discussion on the Financial Consumer Concept Definition [J]. Jurist. 2014(5). 